\title{
Helicity and the Călugăreanu invariant $\dagger$
}

\author{
By H. K. Moffatt and Renzo L. Ricca \\ Department of Applied Mathematics and Theoretical Physics, Silver Street, \\ Cambridge CB3 9EW, U.K.
}

The helicity of a localized solenoidal vector field (i.e. the integrated scalar product of the field and its vector potential) is known to be a conserved quantity under 'frozen field' distortion of the ambient medium. In this paper we present a number of results concerning the helicity of linked and knotted flux tubes, particularly as regards the topological interpretation of helicity in terms of the Gauss linking number and its limiting form (the Călugăreanu invariant). The helicity of a single knotted flux tube is shown to be intimately related to the Călugăreanu invariant and a new and direct derivation of this topological invariant from the invariance of helicity is given. Helicity is decomposed into writhe and twist contributions, the writhe contribution involving the Gauss integral (for definition, see equation (4.8)), which admits interpretation in terms of the sum of signed crossings of the knot, averaged over all projections. Part of the twist contribution is shown to be associated with the torsion of the knot and part with what may be described as 'intrinsic twist' of the field lines in the flux tube around the knot (see equations (5.13) and (5.15)). The generic behaviour associated with the deformation of the knot through a configuration with points of inflexion (points at which the curvature vanishes) is analysed and the role of the twist parameter is discussed. The derivation of the Călugăreanu invariant from first principles of fluid mechanics provides a good demonstration of the relevance of fluid dynamical techniques to topological problems.

\section{Introduction}

The purpose of this paper is to gather together a number of results concerning the helicity $\mathscr{H}$ of an arbitrary solenoidal vector field $\boldsymbol{B}(\boldsymbol{x})$ confined to knotted or linked tube-like structures, particularly as regards its topological interpretation. This interpretation is straightforward when the field $\boldsymbol{B}$ is confined to two linked oriented flux tubes carrying fluxes $\Phi_{1}$ and $\Phi_{2}$ : provided each tube is unknotted and the field lines within each tube are unlinked closed curves circulating once parallel to the tube axis, the helicity is given by

$$
\mathscr{H}=2 n \Phi_{1} \Phi_{2}
$$

where $n$ (an integer, positive, negative or zero) is the (Gauss) linking number of the two tubes (Moreau 1961; Moffatt 1969). If, however, $B$ is confined to a single knotted

$\dagger$ This paper was accepted as a rapid communication.

\$ Present address: Department of Mathematics, University College London, Gower Street, London WC1E 6BT, U.K.

Proc. R. Soc. Lond. A (1992) 439, 411-429

Printed in Great Britain

16
(C) 1992 The Royal Society

Vol. 439. A 
flux tube with flux $\Phi$, then the helicity is related to the topology of the knot in a more subtle way. On purely dimensional grounds, a result of the form

$$
\mathscr{H}=h \Phi^{2},
$$

where $h$ is a real number determined partly by the topology of the knot and partly by the twist of the field $\boldsymbol{B}$ within the knot tube, is to be expected. Since this twist can be arbitrarily prescribed (equivalent to an arbitrary 'framing' of the knot), the number $h$ can take any value, positive or negative. Nevertheless, the fact that $h$ is then invariant under topological deformation of the knot tube and the field within it, does carry some important information about the knot itself.

This type of problem appears to have been first addressed by Călugăreanu (1959, 1961; hereafter referred to as C59 and C61). Călugăreanu considered two neighbouring closed curves $C, C^{*}$ forming the boundaries of a (possibly knotted) ribbon of small spanwise width $\epsilon$, and showed that the linking number $n$ of $C$ and $C^{*}$ can be expressed in the form

$$
n=\mathscr{W}+\mathscr{T}+\mathscr{N}
$$

(this equation is given at the end of p. 613 of $\mathrm{C} 61$ ), where $\mathscr{W}$ and $\mathscr{T}$ are respectively the writhe and the normalized total torsion of $C$ (for definition of these quantities, see (4.8) and (5.13) below), and $\mathscr{N}$ is an integer representing the number of rotations of the unit spanwise vector $N$ on the ribbon relative to the Frenet pair $(\boldsymbol{n}, \boldsymbol{b})$ (unit principal normal and unit binormal) in one passage round $C$. For the moment, we simply note that $\mathscr{T}$ and $\mathscr{N}$ are well defined only if $C$ has no point of inflexion (i.e. no point at which the curvature vanishes). If $C$ is continuously deformed through an inflexional configuration (i.e. through a state that does contain a point of inflexion) then, as will be shown in $\S 6$ below, $\mathscr{T}$ is discontinuous by \pm 1 , but $\mathscr{N}$ is simultaneously discontinuous by an equal and opposite amount $\mp 1$ as a result of the discontinuous behaviour of the Frenet pair $(\boldsymbol{n}, \boldsymbol{b})$ in going through the inflexion, so that the sum $\mathscr{T}+\mathscr{N}$ does vary continuously.

The difficulty associated with inflexion points was recognized by Călugăreanu (see the long footnote on p. 8 of C59) and was to some extent resolved through consideration of a particular example of deformation through an inflexional configuration in C61 (pp. 616-617). Deformations of this type were described as 'degenerate' by Pohl (1968, p. 83); in fact, as pointed out by Ricca \& Moffatt (1992), any deformation whose projection on any plane involves a type I Reidemeister move (see, for example, Kauffman 1991, p. 16) must involve passage through an inflexional configuration. General deformations (or 'ambient isotopies') do therefore typically involve such passage and consideration of the associated behaviour of $\mathscr{T}$ and $\mathscr{N}$ cannot be avoided.

The concept of the self-linking number $S L$ of a closed curve $C$ having no points of vanishing curvature was introduced by Pohl (1968). $S L$ is defined as half the sum of the indices of the cross-tangents of $C$ (i.e. the tangents of $C$ which intersect $C$ in a point distinct from the point of tangency). Pohl showed that $S L$ is an integer, and he proved that

$$
S L=\mathscr{W}+\mathscr{T} .
$$

Under regular isotopy (i.e. continuous deformation of $C$ not passing through any inflexional configuration), $S L$ is invariant so that Pohl's result provides an alternative proof of the invariance under regular isotopy of $\mathscr{W}+\mathscr{T}$, as proved in C59. Pohl's work was extended to higher dimensions by White (1969), who again restricted 
consideration to regular isotopy (which he described (p. 179) as "non-degenerate isotopy').

A third strand of inquiry was introduced by Fuller (1971) who defined the total twist number $T w$ for a ribbon by

$$
T w=\frac{1}{2 \pi} \oint_{C}\left(N^{\prime} \times N\right) \cdot t \mathrm{~d} s
$$

where $N^{\prime}=\mathrm{d} N / \mathrm{d} s$. It is easy to show (see $\S 5$ below) that

$$
T w=\mathscr{T}+\mathcal{N},
$$

(a result nowhere actually stated by Fuller). Fuller then defines the writhe $\mathscr{W}$ through

$$
\mathscr{W}=n-T w,
$$

where $n$ is the linking number of the curves $C, C^{*}$ bounding the ribbon, but nowhere does he prove that this $\mathscr{W}$ is the same as that defined by the Gauss integral (equation (4.8) below). This identification is however established by Călugăreanu's result (1.3) in conjunction with (1.6). The definition (1.5) of Tw provides a quantity which evidently varies continuously under all continuous deformations of the ribbon (i.e. under ambient isotopy).

Curiously, Fuller (1972), in a paper dedicated to Călugăreanu (on his 70th anniversary), gives White (1969) the credit for the result $n=\mathscr{W}+T w$, although this result (with $T w=\mathscr{T}+\mathscr{N}$ ) can be found clearly stated, and coupled with a tentative discussion of the role of inflexion points, in C61. White's achievement was to place this result in the wider context of differentiable manifolds of arbitrary dimension; but the theorem in the form (1.3), or in the equivalent form

$$
n=\mathscr{W}+T w
$$

should surely be described as Călugăreanu's theorem.

We feel it necessary to emphasize this point because in some more recent papers and text books, Călugăreanu is given less than due credit for his achievement. Thus, for example, Pohl (1980) describes (1.8) as 'White's formula', and only rather grudgingly states that 'White's formula was actually put forward by Georges Călugăreanu (1961), originally, for curves $C$ having nowhere vanishing curvature. This proof was very complicated and his formulation confusing...'. We question this judgement and would simply reiterate that Călugăreanu (1961) explicitly considers the zero curvature, or inflexional, problem, whereas White (1969) explicitly excludes such considerations. A general misunderstanding of Călugăreanu's contribution has gradually led people to refer to equation (1.8) as 'White's theorem', so that even in text books (e.g. Kauffman 1987, p. 18; 1991, p. 489), references to C59 and C61 have gradually disappeared.

Our aim in the present paper is to show that all of these results can be obtained in a straightforward manner starting from the helicity invariant of classical fluid dynamics. The link between helicity and the Călugăreanu invariant was conjectured by Moffatt (1981) and was developed on the basis of the result (1.8) by Berger \& Field (1984). However, a direct derivation of (1.8) from the invariance of helicity has not previously been given. We provide such a direct proof in $\$ \S 2-6$ of this paper. First, in $\S 2$, basic results concerning the helicity of linked flux tubes are stated. Then in $\S 3$, it is shown that for a knotted flux tube constructed in such a way that the field lines are closed satellites of $C$, each pair of field lines having linking number $n$, the helicity Proc. R. Soc. Lond. A (1992) 
is given by $\mathscr{H}=n \Phi^{2}$ (the proof was given by Ricca \& Moffatt (1992), but is repeated here for completeness). In $\S 4$, the helicity is decomposed into writhe and twist contributions, the writhe contribution involving the Gauss integral (4.8), which admits interpretation in terms of the sum of signed crossings of $C$ averaged over all projections. In $\S 5$, the twist contribution is considered, part of this being associated with torsion of $C$ and part with what may be described as 'intrinsic twist' of the field lines in the flux tube around $C$. In $\$ 6$ the generic behaviour associated with inflexional configurations is analysed and the role of the twist parameter is discussed in $\S 7$. Finally in $\S 8$ we summarize the conclusions. Our hope is that the alternative proof presented in this paper and the associated discussion may help to demonstrate the relevance of fluid dynamical techniques to topological problems.

\section{The helicity of linked flux tubes}

Consider an arbitrary solenoidal vector field $B(x)=\nabla \times A(x)$ of compact support $\mathscr{D}$ in $\mathbb{R}^{3}$. We suppose that $\boldsymbol{n} \cdot \boldsymbol{B}=0$ on $\partial \mathscr{D}$, the boundary of $\mathscr{D}$. The helicity $\mathscr{H}$ of $\boldsymbol{B}$ is then the pseudo-scalar quantity defined by

$$
\mathscr{H}=\int_{\mathscr{D}} \boldsymbol{A} \cdot \boldsymbol{B} \mathrm{d} V
$$

where $\mathrm{d} V$ is the volume element $\mathrm{d}^{3} \boldsymbol{x}$. Note immediately that $\mathscr{H}$ does not depend on the gauge of $\boldsymbol{A}$; for if $\boldsymbol{A}$ is replaced by $\boldsymbol{A}+\nabla \psi$, then $\mathscr{H}$ is unchanged since

$$
\int_{\mathscr{D}} \boldsymbol{B} \cdot \nabla \psi \mathrm{d} V=\int_{\partial \mathscr{D}} \boldsymbol{n} \cdot \boldsymbol{B} \psi \mathrm{d} S=0
$$

If we adopt the Coulomb gauge for $\boldsymbol{A}$ (i.e. $\nabla \cdot \boldsymbol{A}=0$ ) and impose the further condition $\boldsymbol{A}=O\left(|\boldsymbol{x}|^{-3}\right)$ as $|\boldsymbol{x}| \rightarrow \infty$, then $\boldsymbol{A}(\boldsymbol{x})$ is given by the Biot-Savart law:

$$
A(x)=\frac{1}{4 \pi} \int \frac{B\left(x^{*}\right) \times\left(x-x^{*}\right)}{\left|x-x^{*}\right|^{3}} \mathrm{~d} V^{*}
$$

so that, from (2.1),

$$
\mathscr{H}=\frac{1}{4 \pi} \iint \frac{\left[\boldsymbol{B}(\boldsymbol{x}) \times \boldsymbol{B}\left(\boldsymbol{x}^{*}\right)\right] \cdot\left(\boldsymbol{x}-\boldsymbol{x}^{*}\right)}{\left|\boldsymbol{x}-\boldsymbol{x}^{*}\right|^{3}} \mathrm{~d} V \mathrm{~d} V^{*} .
$$

Consider now the special situation in which $\boldsymbol{B}$ is zero except in two flux filaments centred on two unknotted oriented closed curves $C_{1}, C_{2}$ which may be linked (figure 1). We may suppose that the cross sections of the filaments are small, and that they carry fluxes $\delta \Phi_{1}, \delta \Phi_{2}$. We suppose further that, within each filament, the $\boldsymbol{B}$-lines are themselves unlinked curves which close on themselves after just one passage round the filament, running 'parallel' to $C_{1}, C_{2}$ respectively. In these circumstances, $\mathscr{H}$ may be evaluated directly from (2.1): integrating first over the cross section, $\boldsymbol{B} \mathrm{d} V \rightarrow \delta \Phi_{1} \mathrm{~d} \boldsymbol{x}_{1}, \delta \Phi_{2} \mathrm{~d} \boldsymbol{x}_{2}$ on $C_{1}, C_{2}$ respectively, so that

$$
\mathscr{H}=\delta \Phi_{1} \oint_{C_{1}} A \cdot \mathrm{d} x_{1}+\delta \Phi_{2} \oint_{C_{2}} A \cdot \mathrm{d} x_{2}
$$

Now

$$
\oint_{C_{1}} \boldsymbol{A} \cdot \mathrm{d} \boldsymbol{x}_{1}=\int_{D_{1}} \boldsymbol{B} \cdot \boldsymbol{n} \mathrm{d} S,
$$

Proc. R. Soc. Lond. A (1992) 

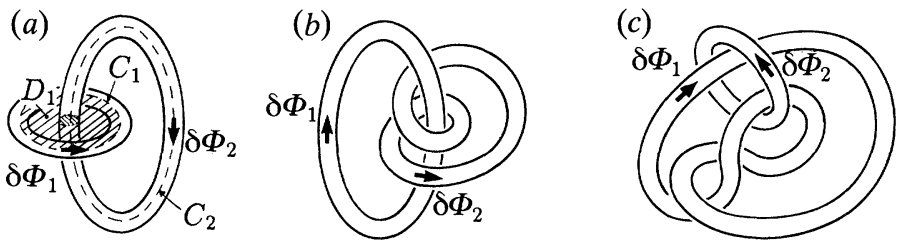

Figure 1. Linked, oriented and unknotted flux tubes with no internal contribution to helicity. In this case $\mathscr{H}=2 n \Phi_{1} \Phi_{2}$, where $n$ is the (Gauss) linking number of the two tube axes. $(a) n=+1$; (b) $n=-2 ;(c) n=0$.

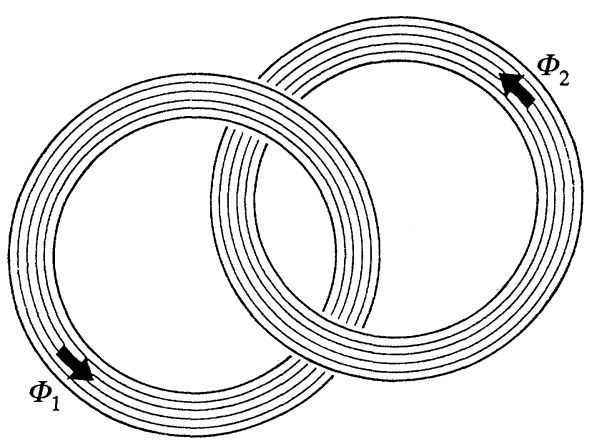

Figure 2

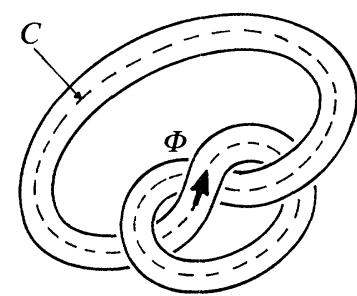

Figure 3

Figure 2. Two linked oriented flux tubes, each one of which is made up of a large number of filaments of small cross section. Each pair of filaments (one from each tube) makes a contribution $2 n \delta \Phi_{1} \delta \Phi_{2}$ to the total helicity, and the total helicity is $2 n \Phi_{1} \Phi_{2}$.

Figure 3. Knotted flux tube whose tube axis is a trefoil knot.

where $D_{1}$ is the open disc surface spanning $C_{1}$. Moreover

$$
\int_{D_{1}} \boldsymbol{B} \cdot \boldsymbol{n} \mathrm{d} S=n \delta \Phi_{2},
$$

where $n$ is the (Gauss) linking number of $\left\{C_{1}, C_{2}\right\}$, i.e. the algebraic number of times that $C_{2}$ crosses $D_{1}$ (allowing for direction of crossing). Three examples are shown in figure 1. Similarly,

and hence, from (2.5),

$$
\oint_{C_{2}} A \cdot \mathrm{d} x_{2}=n \delta \Phi_{1},
$$

$$
\mathscr{H}=2 n \delta \Phi_{1} \delta \Phi_{2} .
$$

Thus $\mathscr{H}$ is determined solely by the two fluxes and the linking number of the two filaments.

In this derivation, it is essential that each flux tube should by itself have zero helicity and this is ensured by the above assumption that the $\boldsymbol{B}$-lines within either tube on its own are unlinked closed curves. In these circumstances also, the value of $n$ is given from (2.4) by integrating over the two cross sections: allowing for the fact that we may have $\boldsymbol{x} \in C_{1}, \boldsymbol{x}^{*} \in C_{2}$ or $\boldsymbol{x} \in C_{2}, \boldsymbol{x}^{*} \in C_{1}$, we find again $\mathscr{H}=2 n \delta \Phi_{1} \delta \Phi_{2}$ with

$$
n=\frac{1}{4 \pi} \oint_{C_{1}} \oint_{C_{2}} \frac{\left(\mathrm{d} x \times \mathrm{d} x^{*}\right) \cdot\left(x-x^{*}\right)}{\left|x-x^{*}\right|^{3}}
$$

This is the well-known Gauss formula for $n$.

Proc. R. Soc. Lond. A (1992) 
The restriction to small cross sections of the two filaments is easily lifted. In the situation indicated by figure 2 in which $\boldsymbol{B}$ is confined to two flux tubes, in each one of which the $\boldsymbol{B}$-lines are again unlinked closed curves passing once (the long way) round the torus, we may regard each flux tube as made up of a large number of filaments of small cross section. Each pair of filaments (one from each tube) make a contribution $2 n \delta \Phi_{1} \delta \Phi_{2}$ to the total helicity, so that summing over all such pairs, this is now given by

$$
\mathscr{H}=2 n \Phi_{1} \Phi_{2} .
$$

\section{The helicity of a single knotted flux tube}

If a flux tube is knotted (as for example in figure 3 ) then its axis $C$ is necessarily a twisted closed curve in $\mathbb{R}^{3}$, and we cannot avoid consideration of the twist of $\boldsymbol{B}$ within the tube itself (which, as will become clear, may change as the flux tube is continuously deformed). It is useful first to define a standard procedure for the construction of a flux tube of prescribed helicity around any given knot $K$. Here we adopt the procedure of Moffatt (1990).

Suppose we deform the knot continuously to lie entirely in the $(x, y)$ plane except at crossing points (the knot being viewed in projection) where we must allow indentations into $z>0$ or $z<0$ (figure 4 ). The crossings are labelled + or according as the overpass must be rotated anticlockwise or clockwise to bring it into coincidence (complete with arrow) with the underpass. By a finite number of crossing switches (i.e. reflections of indentations), it is always possible to convert $K$ to the unknot $K_{0}$ which may then be continuously deformed to the circle $C_{0}: x^{2}+y^{2}=R^{2}$. Conversely, the circle $C_{0}$ may be converted to $K$ by simply reversing these steps (i.e. deformation to $K_{0}$ followed by appropriate crossing switches).

Suppose then that we start with the circle $C_{0}$ and place around this a tubular neighbourhood of small cross section

$$
T_{0}:(r-R)^{2}+z^{2}<(\epsilon R)^{2},
$$

where $r=\left(x^{2}+y^{2}\right)^{\frac{1}{2}}$. Within $T_{0}$, we now define in cylindrical polar coordinates $(r, \theta, z)$, a field

$$
\boldsymbol{B}_{0}=(0,2 \pi r \Phi / V, 0)
$$

where $V=2 \pi^{2} \epsilon^{2} R^{3}$ is the volume of $T_{0}$, and $\Phi$ (as may be easily verified) is the flux of $\boldsymbol{B}_{0}$ through any section of the tube. The field lines of $\boldsymbol{B}_{0}$ are thus unlinked circles near $r=R$. The helicity of the field is clearly zero.

We may now inject helicity (figure 5) by 'Dehn surgery', i.e. by cutting the tube at a section $\theta=$ const., twisting the free ends through a relative angle $2 \pi h$, and reconnecting. We may suppose that the resulting twist is uniformly distributed round the tube. If $h$ is an integer $n_{0}$ say, then each $\boldsymbol{B}$-line in the new flux tube is a closed curve in the form of a helix with axis the circle $r=R$, and each pair of $\boldsymbol{B}$-lines has linking number $n_{0}$. The helicity thus generated is given by

$$
\mathscr{H}_{0}=\int_{0}^{\Phi} 2 n_{0} \phi \mathrm{d} \phi=n_{0} \Phi^{2},
$$

since we may build up the tube by increments $d \phi$, the increment in helicity at each stage being $2 n_{0} \phi \mathrm{d} \phi$, from (2.11).

We now propose to distort $C_{0}$ to the curve $K_{0}$ defined above, carrying the tube $T_{0}$ 


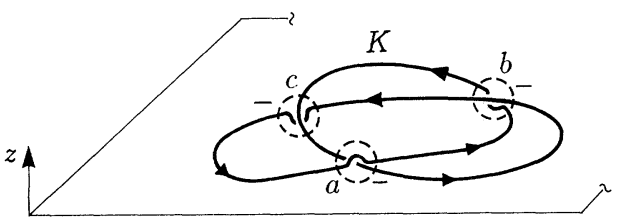

Figure 4

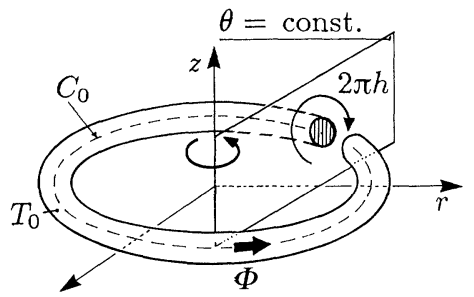

Figure 5

Figure 4. Oriented trefoil knot in a projection plane : at crossing points we must allow indentations into $z>0$ (as at the point $(a)$ in the figure) or $z<0$ (as at points $(b)$ and $(c)$ in the figure).

Figure 5. Dehn surgery: 'cut' the tube at a section $\theta=$ const., 'twist' the free ends through a relative angle $2 \pi h$ and then 'reconnect'.
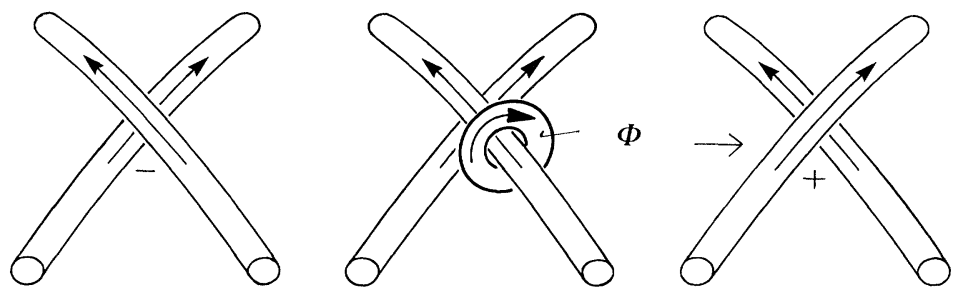

Figure 6. Each negative (positive) switch is equivalent to the insertion of a small loop of flux $\Phi$ annihilating flux on one side of the crossing and creating flux on the other side. In the figure the net increment of helicity is $+2 \Phi^{2}$.

with it. To do this we must specify the isotopy that acts upon the field $\boldsymbol{B}_{\mathbf{0}}$. We picture the field as embedded in an incompressible fluid medium which moves with velocity $\boldsymbol{u}(\boldsymbol{x}, t)$ (where $\boldsymbol{\nabla} \cdot \boldsymbol{u}=0$ ) carrying $\boldsymbol{B}(\boldsymbol{x}, t)$ with it according to the 'frozen field' equation

$$
\partial \boldsymbol{B} / \partial t=\nabla \times(\boldsymbol{u} \times \boldsymbol{B}) .
$$

It is well known that the flux of $\boldsymbol{B}$ through any material surface is conserved under this evolution, and that the helicity of the field is also conserved (Woltjer 1958; Moffatt 1969). We choose a velocity field $\boldsymbol{u}(\boldsymbol{x}, t), t \in\left(-t_{0}, t_{0}\right)$, that brings $C_{0}$ into coincidence with $K_{0}$ and that carries $T_{0}$ into a tubular neighbourhood $T_{0}^{\prime}$ of $K_{0}$. The flux in this tube is then still $\Phi$ and its helicity is still $n_{0} \Phi^{2}$.

We now convert $K_{0}$ to $K$ by appropriate crossing switches. Suppose that $N_{+}$ positive switches (i.e. switches which create positive crossings) and $N_{-}$negative switches are needed to effect this transformation. Each positive switch is equivalent to the insertion of a small loop of flux $\Phi$ annihilating flux on one side of the crossing and creating flux on the other side (figure 6 ). The net increment of helicity is $2 \Phi^{2}$. Similarly a negative switch gives a net increment of helicity $-2 \Phi^{2}$. Hence the helicity of the field in the new tubular neighbourhood $T$ around $K$ is

$$
\mathscr{H}=N \Phi^{2}, \quad N=n_{0}+2\left(N_{+}-N_{-}\right) .
$$

By this construction, the $\boldsymbol{B}$-lines within $T$ are still clearly closed curves, all satellites of $K$, and each pair of $\boldsymbol{B}$-lines having the same linking number $n$, since the crossing switches treat all pairs in the same way. Following the argument of Ricca $\&$ Moffatt (1992), $n$ is in fact equal to $N$; for suppose we divide the flux tube into $m(>1)$ sub-tubes, each sub-tube carrying flux $\Phi_{m}=\Phi / m$ (figure 7 ). The helicity of a 


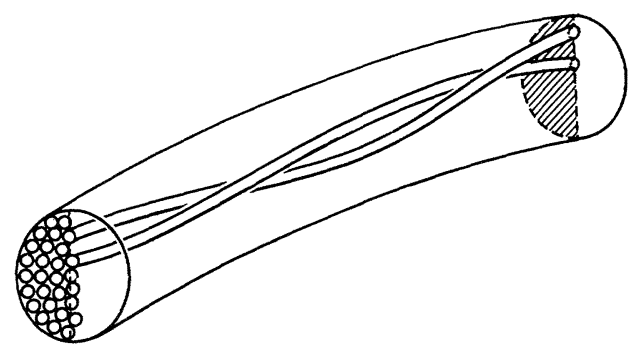

Figure 7. Subdivision of the flux tube into $m(>1)$ sub-tubes.

sub-tube in isolation is $\mathscr{H}_{m}=\mathscr{H} / \mathrm{m}^{2}$, since $\mathscr{H}_{m}$ is quadratically related to the flux $\Phi_{m}$. The total helicity is therefore $m \mathscr{H}_{m}$ plus the sum of the interaction helicities due to linkage of pairs of sub-tubes with linking number $n$, i.e.

$$
\begin{aligned}
\mathscr{H} & =m \mathscr{H}_{m}+\frac{1}{2} m(m-1) \cdot 2 n \Phi_{m}^{2} \\
& =\mathscr{H} / m+(m-1) n \Phi^{2} / m,
\end{aligned}
$$

whence

$$
\mathscr{H}=n \Phi^{2},
$$

so that $n=N$ as asserted.

It is obvious now that the linking number $n$ may be given any desired value by suitable (retrospective) choice of $n_{0}$ :

$$
n_{0}=n-2\left(N_{+}-N_{-}\right) \text {. }
$$

In particular, the choice $n_{0}=-2\left(N_{+}-N_{-}\right)$makes $n=0$, so that the linking number of every pair of $\boldsymbol{B}$-lines in the knotted flux tube is zero. Consideration of the example of figure $1 c$ shows that this does not in general imply that the $\boldsymbol{B}$-lines are unlinked!

\section{The writhe contribution to helicity}

Suppose now that the knot $K$ is in the form of a curve $C$ which has no inflexion points (i.e. points of zero curvature). Let $s$ be arc length on $C$ from some origin $O$, and let the parametric equations of $C$ be $\boldsymbol{x}=\boldsymbol{X}(s)$, where $\boldsymbol{X}(s)$ is periodic with period $L$, the length of $C$. The unit tangent vector is $t=\mathrm{d} X / \mathrm{d} s$, and the unit principal normal $\boldsymbol{n}$ and binormal $\boldsymbol{b}=\boldsymbol{t} \times \boldsymbol{n}$ then satisfy the Frenet equations

$$
\mathrm{d} \boldsymbol{t} / \mathrm{d} s=c \boldsymbol{n}, \quad \mathrm{d} \boldsymbol{n} / \mathrm{d} s=-c \boldsymbol{t}+\tau \boldsymbol{b}, \quad \mathrm{d} \boldsymbol{b} / \mathrm{d} s=-\tau \boldsymbol{n},
$$

where $c(s)$ is the curvature and $\tau(s)$ the torsion of $C$ at position $s$. (Note that $\boldsymbol{n}, \boldsymbol{b}$ and $\tau$ would not be defined at an inflexion point where $c=0$; problems associated with deformation through inflexional configuration will be treated in $\$ 6$ below.)

We now seek to obtain an alternative expression for the helicity in the flux tube $T$ constructed around $K$, directly from the formula (2.1) by considering its limiting behaviour as the cross section of $T$ tends to zero. The field $\boldsymbol{B}$ may be decomposed into the sum of two parts:

$$
B=B_{\mathrm{a}}+B_{\mathrm{m}}
$$

where $\boldsymbol{B}_{\mathrm{a}}$ is the axial field parallel to the tube axis and $\boldsymbol{B}_{\mathrm{m}}$ is the meridional field in meridian planes perpendicular to the tube axis. When the cross section of the tube is small, we may adopt a local cylindrical coordinate system $(r, \theta, z)$ and suppose that

$$
\boldsymbol{B}_{\mathrm{a}}=\left(0,0, B_{z}(r)\right), \quad \boldsymbol{B}_{\mathrm{m}}=\left(0, B_{\theta}(r), 0\right) .
$$


Evidently $\nabla \cdot \boldsymbol{B}_{\mathrm{a}}=0$ and $\nabla \cdot \boldsymbol{B}_{\mathrm{m}}=0$, so that we may introduce separate vector potentials :

$$
\boldsymbol{B}_{\mathrm{a}}=\nabla \times \boldsymbol{A}_{\mathrm{a}}, \quad \boldsymbol{B}_{\mathrm{m}}=\nabla \times \boldsymbol{A}_{\mathrm{m}},
$$

with $\nabla \cdot \boldsymbol{A}_{\mathrm{a}}=0$ and $\nabla \cdot \boldsymbol{A}_{\mathrm{m}}=0$. The lines of force of the $\boldsymbol{B}_{\mathrm{m}}$-field are unlinked circles, so that

$$
\int_{T} \boldsymbol{A}_{\mathrm{m}} \cdot \boldsymbol{B}_{\mathrm{m}} \mathrm{d} V=0
$$

Hence the total field helicity is given by

$$
\begin{aligned}
\mathscr{H} & =\int_{T} \boldsymbol{A}_{\mathrm{a}} \cdot \boldsymbol{B}_{\mathrm{a}} \mathrm{d} V+\int_{T} \boldsymbol{A}_{\mathrm{a}} \cdot \boldsymbol{B}_{\mathrm{m}} \mathrm{d} V+\int_{T} \boldsymbol{A}_{\mathrm{m}} \cdot \boldsymbol{B}_{\mathrm{a}} \mathrm{d} V \\
& =\int_{T} \boldsymbol{A}_{\mathrm{a}} \cdot \boldsymbol{B}_{\mathrm{a}} \mathrm{d} V+2 \int_{T} \boldsymbol{A}_{\mathrm{a}} \cdot \boldsymbol{B}_{\mathrm{m}} \mathrm{d} V
\end{aligned}
$$

(using integration by parts and the divergence theorem) the integrals in each case being over the tube $T$.

Consider first the axial contribution $\mathscr{H}_{\mathrm{a}}=\int_{T} \boldsymbol{A}_{\mathrm{a}} \cdot \boldsymbol{B}_{\mathrm{a}} \mathrm{d} V$. Here we may use the Biot-Savart expression (2.3) in the limiting form

$$
A(x)=\frac{1}{4 \pi} \Phi \oint_{C} \frac{\mathrm{d} x^{*} \times\left(x-x^{*}\right)}{\left|x-x^{*}\right|^{3}}
$$

Although this expression diverges when $\boldsymbol{x} \in C$, its axial component does not diverge, and the limiting expression

$$
\mathscr{H}_{\mathrm{a}}=\frac{1}{4 \pi} \Phi^{2} \oint_{C} \oint_{C} \frac{\left(\mathrm{d} x \times \mathrm{d} x^{*}\right) \cdot\left(x-x^{*}\right)}{\left|x-x^{*}\right|^{3}}=\Phi^{2} \mathscr{W},
$$

say, is finite. The quantity $\mathscr{W}$ is called the writhing number (Fuller 1971) or simply the writhe of $C$, and bears a formal similarity to the Gauss integral (2.10). However, it is important to recognize that $\mathscr{W}$ is not a topological invariant of $C$; in fact it changes continuously (in general) under continuous deformation of $C$.

The physical meaning of the writhe is as follows. Suppose we view the closed curve $C$ projected on a plane with unit normal $\boldsymbol{v}$. We then see a number $n_{+}(\boldsymbol{v})$ of positive crossings and $n_{-}(\boldsymbol{v})$ of negative crossings. Then

$$
\mathscr{W}=\left\langle n_{+}(\boldsymbol{v})-n_{-}(\boldsymbol{v})\right\rangle,
$$

where the angular brackets denote averaging over all directions $\boldsymbol{v}$ of projection. This fact is evident from consideration of the diagram of figure 8 . The elements $\mathrm{d} \boldsymbol{x}, \mathrm{d} \boldsymbol{x} *$ will intersect in projection if and only if $\boldsymbol{n}$ is parallel to $\pm\left(\boldsymbol{r}+\lambda \mathrm{d} \boldsymbol{x}-\mu \mathrm{d} \boldsymbol{x}^{*}\right)$ where $\boldsymbol{r}=\boldsymbol{x}-\boldsymbol{x}^{*}, 0<\lambda<1$ and $0<\mu<1$, i.e. only if $\boldsymbol{v}$ lies within a solid angle

$$
\mathrm{d} \varpi=2\left(\mathrm{~d} \boldsymbol{x} \times \mathrm{d} \boldsymbol{x}^{*}\right) \cdot \boldsymbol{r} / 4 \pi r^{3}
$$

(the factor 2 allowing for the \pm possibilities above). Thus when we average over all directions of $\boldsymbol{v}$, take account of crossing signs and then integrate over all pairs of elements $\mathrm{d} \boldsymbol{x}, \mathrm{d} \boldsymbol{x}^{*}$, we obtain

$$
\mathscr{W}=\frac{1}{4 \pi} \oint_{C} \oint_{C} \frac{\left(\mathrm{d} \boldsymbol{x} \times \mathrm{d} \boldsymbol{x}^{*}\right) \cdot \boldsymbol{r}}{r^{3}}=\left\langle n_{+}(\boldsymbol{v})-n_{-}(\boldsymbol{v})\right\rangle,
$$

Proc. R. Soc. Lond. A (1992) 


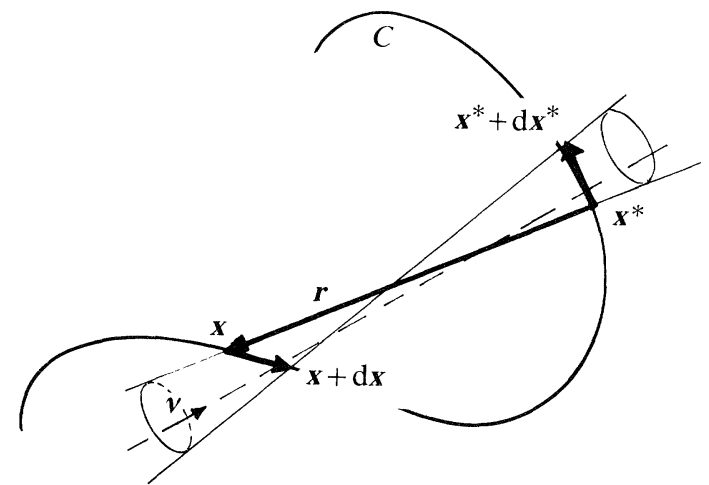

Figure 8. Contribution to the solid angle by elements $\mathrm{d} \boldsymbol{x}, \mathrm{d} \boldsymbol{x} *$ on the curve $C$. The $\boldsymbol{v}$-direction indicates a line of apparent intersection of $\mathrm{d} \boldsymbol{x}, \mathrm{d} \boldsymbol{x}^{*}$.

(a)

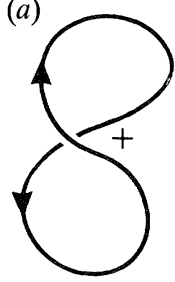

(b)

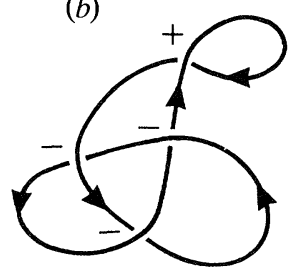

(c)

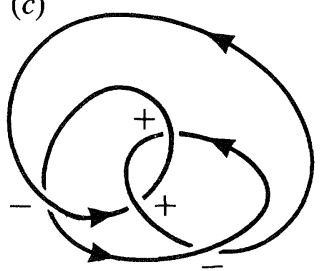

Figure 9. Values of the writhe for a number of flattened configurations. (a) $\mathscr{W}=+1$; (b) $\mathscr{W}=-2 ;(c) \mathscr{W}=0$.

as stated. The geometric interpretation of $\mathscr{W}$ in terms of solid angle was originally discussed in C59 and later, in terms of spherical area, by Fuller (1978); its interpretation in terms of average number of apparent crossings was given by Fuller (1971). The same type of argument leads to Freedman \& He's (1991) expression for the crossing number

$$
\mathscr{C}=\frac{1}{4 \pi} \oint_{C} \oint_{C} \frac{\left|\left(\mathrm{d} \boldsymbol{x} \times \mathrm{d} \boldsymbol{x}^{*}\right) \cdot \boldsymbol{r}\right|}{r_{3}}=\left\langle n_{+}(\boldsymbol{v})+n_{-}(\boldsymbol{v})\right\rangle .
$$

When the knot $K$ is flattened onto the $(x, y)$ plane except for indentations at the crossings, the writhe may be easily computed from the formula (4.11), since in this case $n_{+}(\boldsymbol{v})$ and $n_{-}(\boldsymbol{v})$ are independent of the viewing direction $\boldsymbol{v}$ except for a vanishingly small solid angle of directions nearly parallel to the $(x, y)$ plane. Thus the writhe in this limiting situation is simply given by

$$
\mathscr{W} \sim\left(n_{+}-n_{-}\right)
$$

and is an integer. Values of the writhe for a number of flattened configurations are shown in figure 9 .

We note also that the field $A_{\text {a }}$ provides a family of Seifert surfaces for the knot, as noted by Akhmet'ev \& Ruzmaikin (1992). A Seifert surface is a non-self-intersecting oriented open surface bounded by the knot $K$. Let the cross section of the knot tube $T$ tend to zero. Outside $T, \nabla \times \boldsymbol{A}_{\mathrm{a}}=0$ so there exists a scalar field (not single-valued) such that

$$
A_{\mathrm{a}}=\nabla \Psi_{\mathrm{a}}
$$

and, near $K, \Psi_{\mathrm{a}} \sim(2 \pi)^{-1} \Phi \theta$ where $\theta$ is the azimuth angle used above. Thus the Proc. R. Soc. Lond. A (1992) 
surfaces $\Psi_{\mathrm{a}}=$ const. are all bounded by the knot, and since $\nabla \Psi_{\mathrm{a}}$ is single valued the knot does not intersect such a surface at any other point. Any surface $\Psi_{\mathrm{a}}=$ const. is therefore a Seifert surface.

\section{Torsion and twist contributions to helicity}

Consider now the second contribution in equation (4.6),

$$
\mathscr{H}_{\mathrm{m}}=2 \int_{T} \boldsymbol{A}_{\mathrm{a}} \cdot \boldsymbol{B}_{\mathrm{m}} \mathrm{d} V=2 \int_{T} A_{\theta}(r) B_{\theta}(r) \mathrm{d} V,
$$

arising from the meridional component of the field $\boldsymbol{B}_{\mathrm{m}}$. Note that from the first of (4.3) and the first of (4.4), $\boldsymbol{A}_{\mathrm{a}}=\left(0, A_{\theta}(r), 0\right)$ where

$$
\frac{1}{r} \frac{\mathrm{d}}{\mathrm{d} r}\left(r A_{\theta}\right)=B_{z}(r) \text {. }
$$

Let us consider the change in $\mathscr{H}_{\mathrm{m}}$ under a virtual displacement $\delta \xi(s)$ of the flux tube due to instantaneous changes $\delta c(s), \delta \tau(s)$ in curvature and torsion of $C$. With plane polar coordinates $(r, \theta)$ in the cross-sectional plane at any section $s$ of the tube $T$ (figure 10), with $\theta$ measured from the direction of the principal normal $\boldsymbol{n}$, let

$$
\left.\begin{array}{rl}
\boldsymbol{\xi} & =r \hat{\boldsymbol{e}}_{r}=r(\boldsymbol{n} \cos \theta+\boldsymbol{b} \sin \theta) \\
\hat{\boldsymbol{e}}_{\theta} & =-\boldsymbol{n} \sin \theta+\boldsymbol{b} \cos \theta,
\end{array}\right\}
$$

so that, assuming $\delta \xi$ to be the same for all $(r, \theta)$,

$$
\delta \boldsymbol{\xi}=r \cos \theta \delta \boldsymbol{n}+r \sin \theta \delta \boldsymbol{b},
$$

and so

$$
\frac{\mathrm{d}}{\mathrm{d} s} \boldsymbol{\delta} \boldsymbol{\xi}=(r \cos \theta) \frac{\mathrm{d}}{\mathrm{d} s} \boldsymbol{\delta} \boldsymbol{n}+(r \sin \theta) \frac{\mathrm{d}}{\mathrm{d} s} \delta \boldsymbol{b} .
$$

Since it is only the variation of $\delta \xi$ with arc length $s$ that contributes to distortion of the field, we may suppose that at $s=s_{1}, \delta \boldsymbol{\xi}\left(s_{1}\right)=0$, i.e. $\delta \boldsymbol{n}\left(s_{1}\right)=\delta \boldsymbol{b}\left(s_{1}\right)=0$. Then, from the Frenet relations (4.1), we have

$$
\left.\begin{array}{rl}
\frac{\mathrm{d}}{\mathrm{d} s} \delta \boldsymbol{n} & =-\delta c \boldsymbol{t}+\delta \tau \boldsymbol{b} \\
\frac{\mathrm{d}}{\mathrm{d} s} \delta \boldsymbol{b} & =-\delta \tau \boldsymbol{n}
\end{array}\right\} \text { at } s=s_{\mathbf{1}}
$$

Now under the assumed virtual displacement $\delta \boldsymbol{\xi}(s)$, the axial field $\boldsymbol{B}_{\mathrm{a}}$ (and so $A_{\theta}(r)$ ) is unchanged, but the meridional field $\boldsymbol{B}_{\mathrm{m}}$ at $s=s_{1}$ is changed by an amount

$$
\delta \boldsymbol{B}_{\mathrm{m}}=\left(\boldsymbol{B}_{\mathrm{a}} \cdot \nabla\right) \delta \boldsymbol{\xi}=B_{z}(r) \frac{\mathrm{d}}{\mathrm{d} s} \delta \boldsymbol{\xi},
$$

due to the variation of $\delta \xi$ with arc length (this is the process that in magnetohydrodynamics is known as 'generation of toroidal field by differential rotation' (see Moffatt 1978). Hence, at $s=s_{1}$,

$$
\begin{aligned}
\delta B_{\theta} & =B_{z}(r)\left(\frac{\mathrm{d}}{\mathrm{d} s} \delta \boldsymbol{\xi}\right)_{\theta} \\
& =B_{z}(r)\left[-\sin \theta \boldsymbol{n} \cdot\left(\frac{\mathrm{d}}{\mathrm{d} s} \delta \boldsymbol{\xi}\right)+\cos \theta \boldsymbol{b} \cdot\left(\frac{\mathrm{d}}{\mathrm{d} s} \delta \boldsymbol{\xi}\right)\right] .
\end{aligned}
$$




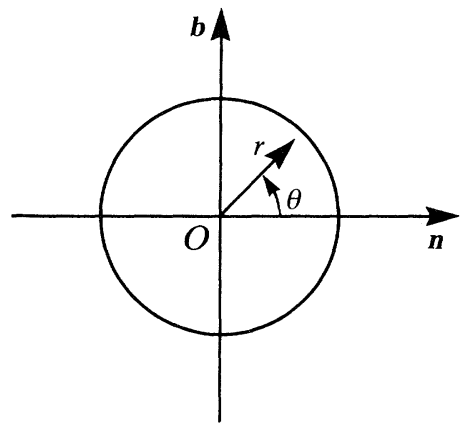

Figure 10. Cross section of the flux tube with plane polar coordinates $(r, \theta)$.

Substituting from (5.5) and (5.6), we have

$$
\delta B_{\theta}=B_{z}(r) r \delta \tau(s) \quad \text { at } \quad s=s_{1} .
$$

Since the same argument may be used at any section, (5.9) gives the field perturbation due to the virtual displacement for all $s_{1}$, and the resulting change in the $\mathscr{H}_{\mathrm{m}}$ is therefore

$$
\begin{aligned}
\delta \mathscr{H}_{\mathrm{m}} & =2 \int_{T} A_{\theta}(r) \delta B_{\theta}(r) \mathrm{d} V \\
& =2 \int_{T} A_{\theta}(r) B_{z}(r) r \delta \tau(s) \mathrm{d} V .
\end{aligned}
$$

If we integrate first over the cross section, using (5.2) and the result

$$
\int_{0}^{\infty} A_{\theta} \cdot \frac{1}{r} \frac{\mathrm{d}}{\mathrm{d} r}\left(r A_{\theta}\right) \cdot r \cdot 2 \pi r \mathrm{~d} r=\frac{1}{2}\left[\left(r A_{\theta}\right)^{2}\right]_{0}^{\infty}=2 \pi \cdot \frac{1}{2} \cdot\left(\frac{\Phi}{2 \pi}\right)^{2},
$$

then from $(5.10)$

$$
\delta \mathscr{H}_{\mathrm{m}}=\Phi^{2} \delta \mathscr{T},
$$

where

$$
\mathscr{T}=\frac{1}{2 \pi} \oint_{C} \tau(s) \mathrm{d} s
$$

is the total torsion of $C$, normalized by the factor $(2 \pi)^{-1}$.

It is easy to show how the total twist number $T w$ defined by (1.5) is related to the normalized total torsion. For this, let us take $N=\boldsymbol{n} \cos \Theta+\boldsymbol{b} \sin \Theta$ as the unit spanwise vector on the ribbon relative to the Frenet pair $(\boldsymbol{n}, \boldsymbol{b})$. By the Frenet equations (4.1), we have

$$
N^{\prime}=\mathrm{d} \boldsymbol{N} / \mathrm{d} s=-c \cos \Theta t+(\tau+\mathrm{d} \Theta / \mathrm{d} s) \hat{\boldsymbol{e}}_{\Theta},
$$

where $\hat{\boldsymbol{e}}_{\Theta}=-\boldsymbol{n} \sin \Theta+\boldsymbol{b} \cos \Theta$. By (1.5), the total twist number for a ribbon is thus given by

$$
T w=\frac{1}{2 \pi} \oint_{C}\left(N^{\prime} \times N\right) \cdot t \mathrm{~d} s=\frac{1}{2 \pi} \oint_{C}\left(\tau+\frac{\mathrm{d} \Theta}{\mathrm{d} s}\right) \mathrm{d} s=\mathscr{T}+\frac{1}{2 \pi}[\Theta]_{C}
$$

and we identify $(1 / 2 \pi)[\Theta]_{C} \equiv \mathscr{N}$. As was pointed out by Banchoff $\&$ White (1975), the total twist number $T w$ depends on the choice of the vector field $N$.

Now, if we consider a time-dependent deformation of $C$ which does not pass through any inflexional configuration, then (5.12) may be written

$$
\mathrm{d} \mathscr{H}_{\mathrm{m}} / \mathrm{d} t=\Phi^{2} \mathrm{~d} \mathscr{T} / \mathrm{d} t
$$

Proc. R. Soc. Lond. A (1992) 
or equivalently

$$
\mathscr{H}_{\mathrm{m}}=\Phi^{2}\left(\mathscr{T}+\mathscr{T}_{0}\right)
$$

where $\mathscr{T}_{0}$ is a constant. In fact $\mathscr{T}_{0}=\mathscr{N}$; however, to establish this point, we have to consider the behaviour when $C$ does pass through an inflexional configuration.

\section{Generic behaviour associated with inflexion points}

If a curve $\boldsymbol{x}=\boldsymbol{X}(s)$ has an inflexion point at $s=s_{\mathrm{c}}$, then $\boldsymbol{t}^{\prime}=\mathrm{d} \boldsymbol{t} / \mathrm{d} s=\mathrm{d}^{2} \boldsymbol{X} / \mathrm{d} s^{2}=0$ at $s=s_{\mathrm{c}}$, so that near $s=s_{\mathrm{c}}$ we have the Taylor expansions

$$
\begin{gathered}
\boldsymbol{t}(s)=\boldsymbol{t}_{\mathrm{c}}+\frac{1}{2}\left(s-s_{\mathrm{c}}\right)^{2} \boldsymbol{t}_{\mathrm{c}}^{\prime \prime}+\ldots \\
\boldsymbol{X}(s)=\boldsymbol{X}_{\mathrm{c}}+\left(s-s_{\mathrm{c}}\right) \boldsymbol{t}_{\mathrm{c}}+\frac{1}{6}\left(s-s_{\mathrm{c}}\right)^{3} \boldsymbol{t}_{\mathrm{c}}^{\prime \prime}+\ldots
\end{gathered}
$$

Moreover, since $|\boldsymbol{t}|=1$,

$$
\left(\boldsymbol{t}^{\prime \prime} \cdot \boldsymbol{t}\right)_{s=s_{\mathrm{c}}}=\left.\frac{\mathrm{d}^{2}}{\mathrm{~d} s^{2}} t^{2}\right|_{s=s_{\mathrm{c}}}=0
$$

so that $\boldsymbol{t}_{\mathrm{c}}^{\prime \prime}$ is perpendicular to $\boldsymbol{t}_{\mathrm{c}}$. We may therefore choose origin at the inflexion point $\left(X_{\mathrm{c}}=0, s_{\mathrm{c}}=0\right)$ and axes $O x y z$ with $O x$ parallel to $\boldsymbol{t}_{\mathrm{c}}$ and $O z$ parallel to $\boldsymbol{t}_{\mathrm{c}}^{\prime \prime}$. The form of the curve near the inflexion point is then given by

$$
\boldsymbol{X}(s)=\left(s, 0, \alpha s^{3}\right),
$$

where $\alpha=\frac{1}{6}\left|\boldsymbol{t}_{\mathrm{c}}^{\prime \prime}\right|$, i.e. it is the plane cubic curve $y=0, z=\alpha x^{3}$. By simple rescaling, we may take $\alpha=1$.

We now wish to consider a time-dependent curve $\boldsymbol{x}=\boldsymbol{X}(s, t)$ passing through the inflexional configuration (6.4) at $t=0$, but having $\partial t / \partial s \neq 0$ when $t \neq 0$. Since

$$
\boldsymbol{t}^{\prime} \cdot \boldsymbol{t}=\frac{1}{2} \partial\left(\boldsymbol{t}^{2}\right) / \partial s=0
$$

we may always, by rigid rotation, ensure that at $s=0, t$ remains parallel to $O x$ and $\boldsymbol{t}^{\prime}$ remains parallel to $O y$. These conditions are satisfied by the time-dependent twisted cubic

for which

$$
X(s, t)=\left(s-\frac{2}{3} t^{2} s^{3}, t s^{2}, s^{3}\right),
$$

and

$$
\begin{aligned}
\boldsymbol{t}=\partial \boldsymbol{X} / \partial s & =\left(1-2 t^{2} s^{2}, 2 t s, 3 s^{2}\right) \\
|\boldsymbol{t}| & =1+O\left(s^{4}\right),
\end{aligned}
$$

so that, near $s=0, t$ is indeed the unit tangent vector. Figure 11 shows this family of curves and their projections on the three coordinate planes.

From (6.7), to leading order in $|t|$ and $|s|$,

so that

$$
\partial \boldsymbol{t} / \partial s \sim 2(0, t, 3 s)
$$

and

$$
\begin{gathered}
c(s, t)=|\partial \boldsymbol{t} / \partial s| \sim 2\left(t^{2}+9 s^{2}\right)^{\frac{1}{2}} \\
\boldsymbol{n}(s, t)=\frac{1}{c} \frac{\partial \boldsymbol{t}}{\partial s} \sim \frac{(0, t, 3 s)}{\left(t^{2}+9 s^{2}\right)^{\frac{1}{2}}} .
\end{gathered}
$$

Note here that for very small $t, \boldsymbol{n}$ rotates through an angle $\pi$ about the direction $\boldsymbol{t}_{\mathrm{c}}=(1,0,0)$ as $s$ increases from $-s_{0}$ to $+s_{0}$ where $s_{0} \gg|t|$; and that this rotation is clockwise (right-handed) for $t<0$, and anticlockwise (left-handed) for $t>0$; thus the number of rotations of the pair $(\boldsymbol{n}, \boldsymbol{b})$ about the tangent direction $\boldsymbol{t}$ in the anticlockwise sense increases by +1 as $t$ increases through zero (at the instant $t=0$, this number is undefined).

Now the binormal is given by $\boldsymbol{b}=\boldsymbol{t} \times \boldsymbol{n}$, and the torsion is obtained from (4.1): for $|t|$ and $|s|$ small,

$$
\tau(s, t) \sim 3 t /\left(t^{2}+9 s^{2}\right) .
$$



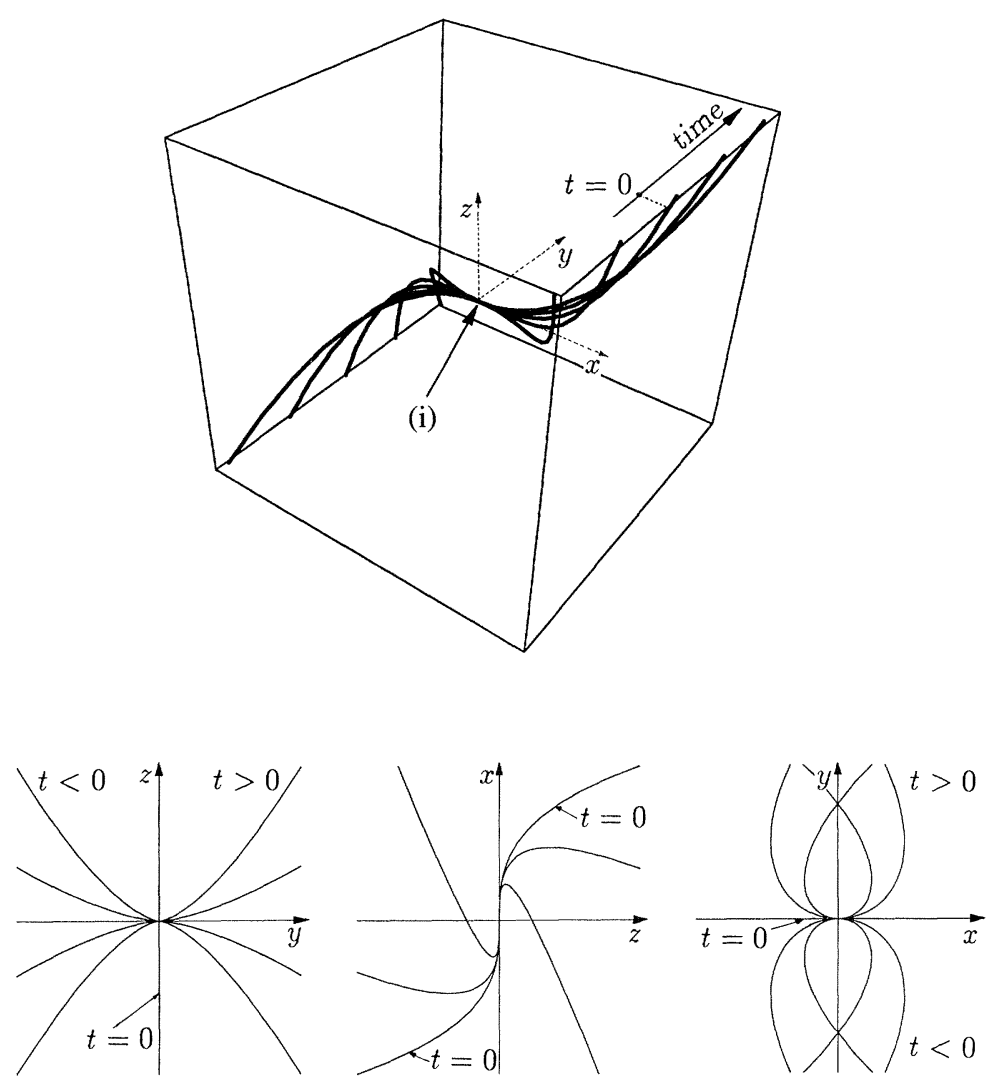

Figure 11. The twisted cubic (6.6) for $-1 \leqslant s \leqslant 1$ and for various values of $t$. The curve contains an inflexion point at (i) $s=0$ when $t=0$. Plane projections are shown below.

As expected, $c$ vanishes only at $t=s=0$, and $\tau$ is singular at this inflexion. However, the singularity is integrable; the contribution to the normalized total torsion $\mathscr{T}$ from any small interval $\left[-s_{0}, s_{0}\right]$ is

$$
\frac{1}{2 \pi} \int_{-s_{0}}^{s_{0}} \tau(s, t) \mathrm{d} s=\frac{1}{\pi} \int_{0}^{s_{0}} \frac{3 t}{t^{2}+9 s^{2}} \mathrm{~d} s=\frac{1}{\pi} \arctan \left(\frac{3 s_{0}}{t}\right),
$$

and, irrespective of the value of $s_{0}$, this jumps from $-\frac{1}{2}$ to $+\frac{1}{2}$ as $t$ increases through zero, i.e. as the curve passes through the inflexional configuration. Hence $\mathscr{T}$ is discontinuous as $C$ passes through the inflexion, with discontinuity $[\mathscr{T}]=+1$. The reverse passage (or equivalently replacement of $t$ by $-t$ in (6.6)) gives a jump $[\mathscr{T}]=-1$. This behaviour, recognized by Călugăreanu (1961) for a particular example, appears to be generic.

\section{Role of the twist parameter}

We have seen from $\$ \S 4$ and 5 above that the helicity of a twisted flux tube with axis $C$ can be expressed in the form

$$
\mathscr{H}=\Phi^{2} h=\Phi^{2}\left(\mathscr{W}+\mathscr{T}+\mathscr{T}_{0}\right) .
$$

Proc. R. Soc. Lond. A (1992) 
(a)

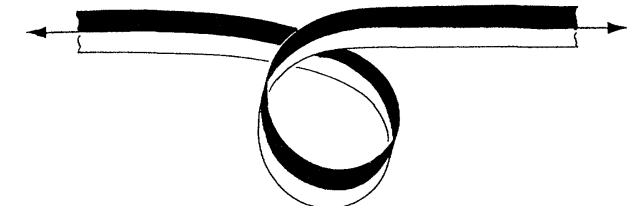

(b)

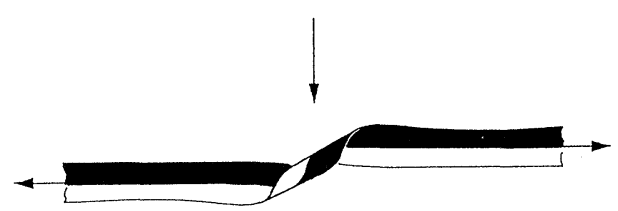

(c)
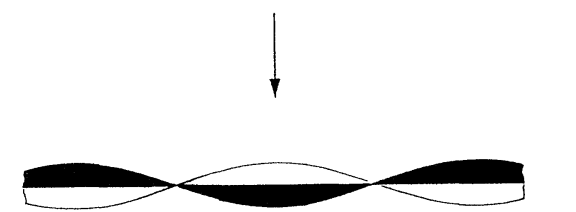

Figure 12. (a) Writhe, $(b)$ torsion and $(c)$ twist contributions of a ribbon to the Călugăreanu invariant. If a coiled ribbon is stretched so that its centre-line becomes straight, then the initial torsion of the centre-line is converted to the final twist of the ribbon about its centre-line.
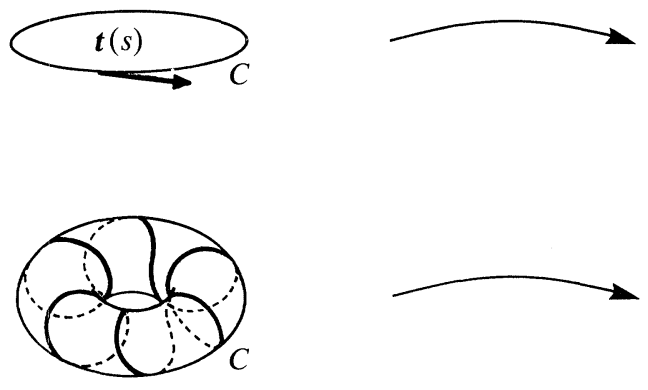

Figure 13. Mapping of closed curves $C$ to their images $C^{\prime}$ on the unit sphere.

Under continuous deformation of the flux tube, $\mathscr{H}$ is conserved and hence

$$
h=\mathscr{W}+\mathscr{T}+\mathscr{T}_{0}=\text { const. }
$$

The writhe $\mathscr{W}$ defined by (4.8) varies continuously as $C$ is deformed continuously; however, if $C$ passes through an inflexional configuration, then $\mathscr{T}$ jumps by \pm 1 . Hence, as $\mathscr{T}$ jumps by \pm 1 , the term $\mathscr{T}_{0}$ must jump by a compensating amount $\mp 1$, to maintain the overriding invariance of helicity.

The equal and opposite jumps in $(\mathscr{W}+\mathscr{T})$ and $\mathscr{T}_{0}$ may be understood with reference to the simple example illustrated in figure 12. If a coiled ribbon is stretched so that its centre-line becomes straight (at which stage $\mathrm{d} t / \mathrm{d} s \equiv 0$ on the centre-line!), then the initial writhe of the centre-line is converted to the final twist of the ribbon about its centre-line. This example is not generic since it involves the appearance of a continuum of inflexion points. However, it captures the essence of the nature of the interchange between $(\mathscr{W}+\mathscr{T})$ and $\mathscr{T}_{0}: \mathscr{T}_{0}$ represents the intrinsic twist of the ribbon about its centre-line, and this in general jumps by $\mp 1$ when the centre-line is deformed through an isolated inflexion point. 

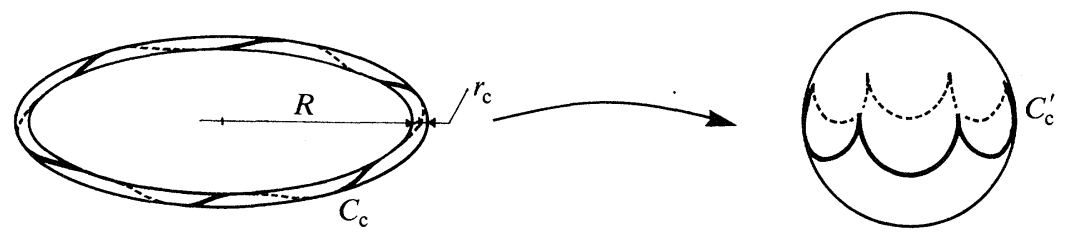

Figure 14. Mapping of the curve $C_{\mathrm{c}}$ on a 'critical' torus to its image $C_{\mathrm{c}}^{\prime}$ on the unit sphere. At the critical value $r_{\mathrm{c}}=R / \mathrm{m}^{2}$, inflexion points appear on $C_{\mathrm{c}}$ and they are mapped to the $m$ cusps on the unit sphere $(m=6$ in the case illustrated).

There is a further useful way of picturing this inflexional behaviour (see figure 13); the set of unit vectors $\boldsymbol{t}(s)$ on a closed curve $C$ trace out a corresponding closed curve $C^{\prime}$ on the unit sphere. For example a circle $C$ corresponds to an equatorial circle $C^{\prime}$; a helix wound around a fat torus corresponds to an epicyclic curve with a number of double points, and so on. If $C$ is continuously deformed, then its image $C^{\prime}$ is continuously deformed also, and the number of double points of $C^{\prime}$ may change. In fact this does happen when $C$ is deformed through an inflexion (at $s=s_{1}$ say) at which $\mathrm{d} t / \mathrm{d} s=0$. When $C$ has an inflexion, $C^{\prime}$ has a cusp at the corresponding point on the sphere.

The case of a helix wound around a torus,

$$
X(s)=\left[\left(R+r_{0} \cos m \phi\right) \cos \phi,\left(R+r_{0} \cos m \phi\right) \sin \phi, r_{0} \sin m \phi\right],
$$

(where $m$ is an integer) is particularly interesting in this respect (figure 14). If $r_{0}=0$, this is a circle, the principal normal $\boldsymbol{n}=\boldsymbol{n}_{0}$ pointing towards its centre. For very small values of $r_{0}$, the direction of $\boldsymbol{n}$ simply oscillates about the position $\boldsymbol{n}_{0}$ as we move round $C$. As $r_{0}$ increases further the amplitude of these oscillations increases, until at a critical value $r_{\mathrm{c}}\left(=R / \mathrm{m}^{2}\right)$ inflexion points appear on $C$ at the points where $\cos m \phi=-1$; for $r_{0}>r_{\mathrm{c}}$ the principal normal $\boldsymbol{n}$ makes $m$ complete rotations around the axis of the torus in one passage round $C$.

If we now place a flux tube of cross-sectional radius $\epsilon \ll r_{\mathrm{c}}$ around $C$, and consider a time-dependent deformation in which $r_{0}=r_{0}(t)$ decreases through the critical value $r_{\mathrm{c}}$, then $[\mathscr{T}]=-m,\left[\mathscr{T}_{0}\right]=+m$ in going through the critical point, i.e. torsional helicity is instantaneously converted to twist helicity, the total helicity being of course conserved.

The fact that $\mathscr{T}_{0}$ jumps by \pm 1 whenever $C$ passes through a single inflexion suggests the interpretation that it represents, in some sense, the number of rotations of the flux tube (or of the associated set of ribbons) about its axis in one passage around $C$. This concept is, however, quite elusive, because one must specify carefully the frame of reference with respect to which the flux tube rotates. There is no difficulty in this when $C$ is not in an inflexional configuration, for then we may use the Frenet frame $(\boldsymbol{t}, \boldsymbol{n}, \boldsymbol{b})$. Let $C, C^{*}$ be two neighbouring $\boldsymbol{B}$-lines in the flux tube (the boundaries of a ribbon), and, as before, let $N(s)$ be the spanwise vector from $C$ to $C^{*}$ on this ribbon. Let $\mathscr{N}$ be the (integer) number of rotations of $N(s)$ about $t$ with respect to the Frenet frame (as defined in $\S 5$ ). We shall show that in fact $\mathscr{T}_{0}=\mathscr{N}$.

Under arbitrary continuous deformations of the flux tube, $N(s, t)$ is a continuous vector function of $(s, t)$, and its components with respect to a fixed cartesian frame of reference are also continuous. However, if $C$ passes through an inflexion at $s_{\mathrm{c}}$, then, as we have seen in $\S 6$, the number of rotations of the Frenet pair $(\boldsymbol{n}, \boldsymbol{b})$ about the tangent vector $t$ in one passage round $C$ changes by \pm 1 ; hence the number $\mathscr{N}$ of rotations of $N(s, t)$ relative to the Frenet frame changes by \pm 1 .

Proc. R. Soc. Lond. A (1992) 


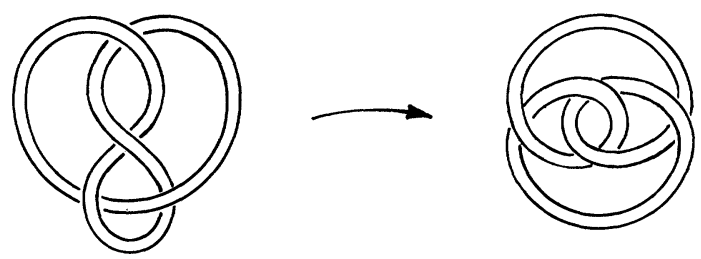

Figure 15. A plane projection of a knot may always be arranged so to have no inflexion points. Here the figure-of-eight knot, with two inflexions, is isotoped to a configuration with no inflexion points.

The knot $K$ and its associated flux tube may always be deformed to lie nearly in the $(x, y)$ plane (as in $\S 3$ ) so that the write $\mathscr{W}$ is (in the limit) an integer $\left(n_{+}-n_{-}\right)$. We may also always arrange that this plane projection of $K$ has no inflexion points; for example the figure-of-eight, containing two prototype inflexions, can be isotoped (figure 15) to a curve of non-vanishing curvature. In such a 'standard' configuration, the torsion $\tau(s)$ is zero everywhere except in the small indentations where it is small; hence in the plane limit, $\mathscr{T}=0$. In this configuration therefore the helicity is given by

so that

$$
\mathscr{H}=n \Phi^{2}=\left(n_{+}-n_{-}\right) \Phi^{2}+\mathscr{T}_{0} \Phi^{2}
$$

is an integer. Consideration of the special case of a circle $\left(n_{+}=n_{-}=0\right)$ shows that $\mathscr{T}_{0}$ is indeed the number of twists of the ribbon (unambiguous in the plane configuration) about its centre-line. We thus identify $\mathscr{T}_{0}$ and $\mathscr{N}$ in this standard configuration, and hence in every configuration of the knot.

\section{Summary}

In this paper we have discussed several properties of the helicity of linked and knotted flux tubes. Different contributions to helicity have been analysed in terms of the Gauss linking number (§2) and in terms of the Călugăreanu invariant (1.3). If the field lines in a single knotted flux tube are twisted closed curves which close on themselves after one passage around the tube, then the helicity of the flux tube is given by

$$
\mathscr{H}=\int_{T} \boldsymbol{A} \cdot \boldsymbol{B} \mathrm{d} V=n \Phi^{2},
$$

where $\Phi$ is the flux associated with the tube (§3). The integer $n$ is an invariant under frozen field distortion of the tube, and is identified with the Călugăreanu invariant (1.3). We have demonstrated this invariance by starting from the known invariance of helicity. The helicity has been decomposed into writhe and twist contributions, the writhe contribution involving the Gauss integral, which admits interpretation in terms of the sum of signed crossings of the knot averaged over all projections ( $\$ 4)$. Part of the twist contribution is shown to be associated with the torsion of the knot and part with what may be described as 'intrinsic twist' of the field lines in the flux tube around the knot ( $\$ 5)$. The generic behaviour associated with the deformation of the knot through a configuration with points of inflexion (points at which the curvature vanishes) has been analysed $(\S 6)$ and the role of the twist parameter has been discussed $(\$ 7)$.

Proc. R. Soc. Lond. A (1992) 
In deriving the relation

$$
\mathscr{H}=n \Phi^{2}=(\mathscr{W}+\mathscr{T}+\mathscr{N}) \Phi^{2},
$$

where the writhe $\mathscr{W}$ is defined by equation (4.8), the normalized total torsion $\mathscr{T}$ is defined by equation (5.13) and $\mathscr{N}=\mathscr{T}_{0}$ is the twist parameter, we have shown that (generically) $(\mathscr{W}+\mathscr{T})$ jumps discontinuously through \pm 1 as $C$ passes through an inflexional configuration and that by virtue of the invariance of $\mathscr{H}$, there is then a compensating jump of $\mp 1$ in $\mathscr{N}$. This behaviour has been previously recognized by Ricca \& Moffatt (1992) and is associated with the classical type I Reidemeister move of ambient isotopies.

The Călugăreanu invariant is fundamental in relation to many problems that involve continuous deformation of tube-like structures. Examples in the literature range from the theory of dynamical systems (Uezu 1990) to the biochemistry of excitable media (Winfree 1990), from the quantum field theory of string-like objects (Tze \& Nam 1989) to studies of DNA coiling ('Tsuru \& Wadati 1986), from the theory of propagation of spinning particles (Jaroszewicz \& Kurzepa 1991) to the general problem of protein folding (De Santis et al. 1986). Fundamental topological aspects of these phenomena can be successfully described in terms of the Călugăreanu invariant. In this paper we hope that the direct derivation of the Călugăreanu invariant from first principles of fluid mechanics together with the discussion of the generic behaviour associated with inflexional configurations, which are ubiquitous in many processes of continuous deformation of field structures, provides a good demonstration of the relevance of fluid dynamical techniques to topological problems.

We thank Dr J. S. Langer, Director of the Institute for Theoretical Physics at UCSB (which is supported by NSF Grant PHY89-04035), for his kind hospitality at the Institute, where this work was initiated. One of us (R.L.R.) acknowledges the financial support from Associazione Sviluppo Scientifico e Tecnologico del Piemonte (Turin, Italy).

\section{References}

Akhmet'ev, P. \& Ruzmaikin, A. 1992 Borromeanism and bordism. In Topological aspects of the dynamics of fluids and plasmas (ed. H.K. Moffatt et al.) pp. 249-264. Dordrecht: Kluwer.

Banchoff, T. F. \& White, J. H. 1975 The behaviour of the total twist and self-linking number of a closed space curve under inversions. Math. Scand. 36, 254-262.

Berger, M. A. \& Field, G. B. 1984 The topological properties of magnetic helicity. J. Fluid Mech. 147, $133-148$.

Călugăreanu, G. 1959 L'intégral de Gauss et l'analyse des noeuds tridimensionnels. Rev. Math. pures appl. 4, 5-20. (C59 in text.)

Călugăreanu, G. 1961 Sur les classes d'isotopie des noeuds tridimensionnels et leurs invariants. Czechoslovak Math. J. 11, 588-625. (C61 in text.)

De Santis, P., Palleschi, A. \& Chiavarini, S. 1986 Topological approach to protein folding. Gazz. Chim. It. 116, 561-567.

Freedman, M. H. \& He, Z.-X. 1991 Divergence-free fields: Energy and asymptotic crossing number. Ann. Math. 134, 189-229.

Fuller, F. B. 1971 The writhing number of a space curve. Proc. natn. Acad. Sci. U.S.A. 68, 815-819.

Fuller, F. B. 1972 How the writhing number of a curve depends on the curve. Rev. Roum. Math. pures appl. 17, 1329-1334.

Fuller, F. B. 1978 Decomposition of the linking of a closed ribbon: a problem from molecular biology. Proc. natn. Acad. Sci. U.S.A. 75, 3557-3561.

Proc. R. Soc. Lond. A (1992) 
Jaroszewicz, T. \& Kurzepa, P. S. 1991 Spin, statistics and geometry of random walks. Ann. Phys. 210, 255-322.

Kauffman, L. H. 1987 On knots. Princeton University Press.

Kauffman, L. H. 1991 Knots and physics. World Scientific Publishing.

Moffatt, H. K. 1969 The degree of knottedness of tangled vortex lines. J. Fluid Mech. 35, 117-129.

Moffatt, H. K. 1978 Magnetic field generation in electrically conducting fluids. Cambridge University Press.

Moffatt, H. K. 1981 Some developments in the theory of turbulence. J. Fluid Mech 106, 27-47.

Moffatt, H. K. 1990 The energy spectrum of knots and links. Nature, Lond. 347, 367-369.

Moreau, J. J. 1961 Constantes d'un îlot tourbillonnaire en fluid parfait barotrope. C.r. hebd. Sêanc. Acad. Sci., Paris 252, 2810-2812.

Pohl, W. F. 1968 The self-linking number of a closed space curve. J. Math. Mech. 17, 975-985.

Pohl, W. F. 1980 DNA and differential geometry. Math. Intelligencer 3, 20-27.

Ricca, R. L. \& Moffatt, H. K. 1992 The helicity of a knotted vortex filament. In Topological aspects of the dynamics of fluids and plasmas (ed. H. K. Moffatt et al.) pp. 225-236. Dordrecht: Kluwer.

Tsuru, H. \& Wadati, M. 1986 Elastic model of highly supercoiled DNA. Biopolymers 25, 2083-2096.

Tze, C.-H. \& Nam, S. 1989 Topological phase entanglements of membrane solitons in division algebra sigma models with a Hopf term. Ann. Phys. 193, 419-471.

Uezu, T. 1990 Topological structure in flow systems. Prog. theor. Phys. 83, 850-874.

White, J. H. 1969 Self-linking and the Gauss integral in higher dimensions. Am. J.Math.91, 693-728.

Winfree, A. T. 1990 Stable particle-like solutions to the nonlinear wave equations of threedimensional excitable media. SIAM Rev. 32, 1-53.

Woltjer, L. 1958 A theorem on force-free magnetic fields. Proc. natn. Acad. Sci. U.S.A. 44, 489-491. 